\title{
Humour et transgression dans la littérature orale d'une société de Nouvelle-Guinée
}

Bernard Juillerat

\section{(2) OpenEdition}

1 Journals

Édition électronique

URL : https://journals.openedition.org/clo/721

DOI : $10.4000 /$ clo. 721

ISSN : 2266-1816

Éditeur

INALCO

Édition imprimée

Date de publication : 1 janvier 2009

Pagination : 165-183

ISBN : 978-2-85831-188-0

ISSN : 0396-891X

Référence électronique

Bernard Juillerat, « Humour et transgression dans la littérature orale d'une société de Nouvelle-

Guinée », Cahiers de littérature orale [En ligne], 66 | 2009, mis en ligne le 25 février 2013, consulté le 02 juillet 2021. URL : http://journals.openedition.org/clo/721 ; DOI : https://doi.org/10.4000/clo.721

Ce document a été généré automatiquement le 2 juillet 2021.

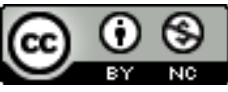

Cahiers de littérature orale est mis à disposition selon les termes de la Licence Creative Commons Attribution - Pas d'Utilisation Commerciale 4.0 International. 


\title{
Humour et transgression dans la littérature orale d'une société de Nouvelle-Guinée
}

\author{
Bernard Juillerat
}

\section{NOTE DE L'ÉDITEUR}

Article paru pour la première fois dans Cahiers de littérature orale, $\mathrm{n}^{\circ} 8,1980$, p. 125-149.

1 Ouvrir à nouveau le dossier du sens et des mécanismes de l'humour dans les sociétés diverses ou à des époques différentes, c'est reposer entre autres le problème de l'universalité des structures du comique en se posant des questions du genre: rit-on partout des mêmes choses? Ou comment expliquer que ce qui fait beaucoup rire dans une culture donnée laisse l'étranger de passage presque indifférent ? Le but du présent article n'est pas d'argumenter une fois de plus sur un sujet à propos duquel il est apparu évident que la réponse était d'ordre culturel, chaque expression du comique renvoyant au plan du contenu à des références sociales ou idéologiques précises sans la connaissance desquelles le point d'articulation central du comique risque de passer inaperçu; en deçà de la culture globale d'une société, il y a en outre des variations internes dues aux différences de classes, d'éducation et, évidemment, à la personnalité et à la suggestibilité des individus. L'humour renvoie à des nuances idéologiques ou à des habitudes sociales précises qui, par leur spécificité culturelle, s'opposent au caractère beaucoup plus universel de la forme ou des mécanismes du comique, qui est souvent engendré par ce petit "décalage», ce «défaut", ce "désordre » structurel (cette « disjonction " pour reprendre le terme de V. Morin, 1966) surgissant soudain au milieu d'une séquence narrative, dont la logique se trouve ainsi bouleversée. Nous allons voir que le déplacement le plus fréquemment rencontré dans l'humour de la société prise en considération ici est de l'ordre de la transgression, sémantique et ethnicosociale : le contenu du tabou fournit alors le sens et renvoie à la culture, tandis que la transgression constitue une forme de comportement universellement reconnue. 
Le rire n'est que l'une des réactions possibles engendrées par cette alliance hasardeuse du contenu et de la forme: dans la vie sociale réelle, une transgression peut avoir des conséquences graves et déboucher sur le tragique ou la violence, mais le même événement apparaissant dans un conte se trouve dédramatisé par l'imaginaire, et le tragique ainsi évacué fait place à la détente.

2 Ce sont à la fois cette spécificité du contenu culturel et cette universalité de la forme que nous allons retrouver dans les épisodes ci-dessous, extraits des mythes d'une société de Nouvelle-Guinée.

\section{LA LITTÉRATURE ORALE ERI}

3 Les Eri (ou Amanab) constituent un groupe d'environ trois mille cinq cents personnes parlant le mô, une langue non austronésienne; ils habitent de petits hameaux pluriclaniques (divisés en moitiés chez les Eri septentrionaux et centraux) et vivent d'horticulture itinérante forestière, de chasse, de cueillette et d'un petit élevage intermittent de porcs.

4 Leur littérature ${ }^{1}$ orale contient en filigrane l'essentiel de leurs préoccupations idéologiques et reflète notamment leurs conceptions sur la fertilité et la reproduction $\mathrm{du}$ monde naturel et des hommes, ainsi que les rapports sociaux, et plus particulièrement les relations entre les sexes et entre les proches parents. On peut distinguer trois catégories littéraires :

5 1. Le mythe de la création de l'univers, représentant une connaissance ésotérique interdite aux femmes.

6 2. Des mythes non secrets, relatant des événements postérieurs à la création originelle et décrivant en particulier les faits et gestes des deux héros civilisateurs Wefroog et Abunung, leurs rencontres avec les esprits sylvestres et les premiers humains.

7 3. Des histoires relatant la rencontre inopportune d'humains avec des fantômes ou d'autres esprits malfaisants.

8 Le premier type de récit est trop sacré pour contenir la moindre note humoristique, et en rire équivaudrait à une sorte de blasphème. Ce mythe cosmogonique n'a d'ailleurs jamais une fonction de diversion ; il n'est qu'une connaissance mise « en archive » dans la mémoire de certains hommes qui, pour ne pas l'oublier, le verbalisent occasionnellement entre eux et en secret avant de le transmettre à un successeur de leur choix à la génération suivante. Cette connaissance est profondément liée au rituel, dont elle fournit le principe explicatif. Par contre, les deux autres catégories de textes oraux comportent de nombreux éléments humoristiques disséminés dans l'ensemble $\mathrm{du}$ corpus. Aucune histoire en particulier n'est conçue comme spécifiquement comique, sauf peut-être quelques récits relatant les combats, poursuites et tromperies entre le héros civilisateur Wefroog et les esprits féminins sawango. Ces personnages appartiennent au monde sacré (et, dans d'autres contextes, seraient pris très au sérieux), mais le cocasse des situations désacralise le récit (voir les exemples 1 à 3,13 et 16, ci-dessous). C'est pourquoi - conjointement avec les histoires de fantômes qui, elles, ne renvoient pas aux temps primordiaux - ce sont là des premières narrations que l'on accorde à l'ethnologue. Par la suite, il s'entendra raconter les mythes non ésotériques où l'élément humoristique ou récréatif est encore important. Quant au récit cosmogonique, secret et sacré, de notre première catégorie, il ne saurait en principe 
être communiqué à l'étranger. La proportion d'humour augmente donc en fonction inverse de la proportion de secrets contenue dans les textes. Et plus les textes contiennent de ces éléments comiques, plus fréquemment ils seront narrés. Le mythe sérieux et ésotérique (sacré) exprime un haut degré de connaissance, mais n'offre qu'un faible rendement sur le plan de la communication sociale, alors que le récit comique (profane) a une valeur cognitive faible, mais une fonction socialisante essentielle.

Dans la tribu eri où nous avons travaillé, les récits (catégories 2 et 3 ) étaient rarement racontés et c'est surtout à l'occasion de l'enquête que beaucoup de jeunes ont pu entendre, souvent pour la première fois, les mythes et contes de leur tradition littéraire. Le conteur, généralement un homme d'âge mûr, racontait son histoire avec sérieux, mais en l'agrémentant d'intonations et d'onomatopées expressives, parfois de gestes, devant un auditoire restreint d'enfants et d'adolescents réagissant aux péripéties du récit par des exclamations, des onomatopées et, bien sûr, des rires. Parfois un autre adulte présent ajoutait quelques détails ou devançait le narrateur pour lui « voler » le plaisir de relater la fin d'un épisode. À l'écart, en dehors de la maison (souvent sous les pilotis si le groupe s'était installé chez l'ethnologue) quelques femmes écoutaient aussi, plus discrètes, en étouffant tant bien que mal leurs rires.

\section{LES ARTICULATIONS DE L'HUMOUR}

En parlant d'humour ou de comique dans le cadre d'une société étrangère, il est nécessaire d'en préciser les degrés et de dire sous quelles formes cet humour se manifeste, comment le rire éclate sans gêne ou au contraire peut être retenu et intériorisé. C'est là encore autant la forme que le contenu qui interviennent dans leur rencontre pour donner à l'humour son éclat sonore dans la farce ou le quiproquo, ou au contraire pour le réfréner jusqu'à un silence ambigu face à la transgression des interdits les plus stricts; ceux par exemple qui touchent à la procréation, et où la transgression peut consister à confondre la catégorie des substances puissantes et dangereuses (comme le sang menstruel) avec celle des produits alimentaires (voir exemple 26). Le sens profond est ici trop lié au sacré pour que le rire se manifeste et tout le plaisir consiste à savourer silencieusement, en deçà du comique proprement dit, la richesse symbolique et l'ambivalence d'une transgression aussi osée.

11 L'examen des épisodes comiques dans la littérature eri nous a amené d'abord à distinguer un comique non linguistique d'un comique fondé sur la langue. Nous ne traiterons ici que de la première catégorie qui est de loin la plus importante, la seconde se limitant à quelques jeux onomatopéiques et à des déformations phonologiques dans la bouche de certains personnages. Le comique non linguistique nous a paru s'articuler autour de quatre types de dispositifs (que nous hésitons à qualifier de procédés), qui d'ailleurs n'apparaissent jamais isolément, mais toujours conjointement avec un ou deux autres. Ce sont :

- la transgression sémantique par métaphore ou métonymie,

- le comique d'antagonisme : duperies, quiproquo, etc.,

- le procédé narratif : comique de répétition,

- le domaine privilégié : parenté, sexualité, pollution, alimentation.

12 Le procédé comique le plus fréquent va dès lors consister à introduire la transgression dans l'un ou plusieurs de ces domaines pour y brouiller les catégories instaurées par la culture. Derrière la transgression sémantique (métaphore ou métonymie) est alors 
perçue une transgression sociale ou morale. Les domaines privilégiés cités ci-dessus se prêtent tout particulièrement à la transgression, car plus que d'autres ils se trouvent régis par des règles sociales strictes ou même, pour le dernier au moins, par des règles naturelles: produits consommables ou inconsommables. L'alimentation notamment débouche, par le biais de la transgression, sur des aberrations de comportement particulièrement fortes aux yeux des Eri, comme l'anthropophagie ou la scatophagie. Avant l'alimentation déviante, il y a le simple contact corporel ou la proximité des substances polluantes qui constitue un premier niveau de transgression. L'épisode résumé plus bas (26) montre bien les différents degrés de proximité par lesquels passe successivement le personnage.

\section{LA TRANSgRESSION SÉMANTIQUE}

13 Précisons tout de suite que par la transgression sémantique, il ne faut pas entendre ici le changement de sens d'un mot (il s'agirait alors d'un procédé linguistique), mais une modification dans l'usage culturel normal d'un objet ou dans le rôle social normal d'un individu. Il s'agit dans le premier cas (objet détourné de son usage habituel) d'une transgression technologique, dans le second cas (statut social non respecté) d'une transgression sociale. Mais nous incluons les deux phénomènes dans la même catégorie de "transgression sémantique ", car il s'agit du même procédé : faire jouer à un objet, un animal ou une personne une fonction qui n'est pas la sienne, le détourner de sa destinée habituelle, bref lui donner un «sens culturel» inattendu. Dans tous les exemples qui vont être donnés, la transgression nous paraît d'ordre sémantique, car il y a entre l'objet (ou la personne) et sa fonction culturelle normale la même relation qui lie un signifiant à un signifié. La transgression peut prendre la forme du remplacement pur et simple du sens habituel par un autre (c'est ce que nous appelons « déplacement sémantique par métaphore ») ou bien elle peut prendre la forme d'un " déplacement sémantique par métonymie", où c'est une partie qui joue le rôle du tout. On remarquera d'autre part que la "disjonction ", qui est la source du comique, a toujours lieu au niveau des comportements, pas à celui des discours, contrairement à ce qui caractérise généralement nos « histoires » occidentales.

\section{DÉPLACEMENTS MÉTAPHORIQUES}

Examinons les épisodes suivants, extraits de différents textes et considérés comme humoristiques par les Eri :

1. Wefroog et un génie femelle se disputent un fruit de pandanus. Ils finissent par en venir aux mains et se frappent avec le fruit, puis ôtent leurs ceintures et s'en frappent également.

2. Pendant l'absence du génie, Wefroog vole les fruits de pandanus et laisse ses excréments à la place.

3. Un génie femelle a dévoré l'enfant de Wefroog. Celui-ci se venge en lui faisant avaler de l'eau bouillante et des pierres chauffées à blanc.

4. Une vieille femme trouve une calebasse (dont on fait les étuis péniens) et l'attache à sa jupe de cordelettes devant son pubis.

5. Wefroog trouve son pénis trop long et le lime en l'aiguisant sur une meule dormante.

6. Une femme propose à un homme de faire l'amour. Celui-ci fait semblant d'accepter et introduit dans le sexe de la femme un fruit urticant.

7. Un chasseur tue un cochon sauvage, mais au lieu de le rapporter à sa famille, il le 
dépose sur une plate-forme funéraire.

8. La veuve de Wefroog dit à Abunung: "Si tu veux m'épouser, va tuer un homme ». Abunung part, tire une flèche contre un arbre à sève rougeâtre, puis prétend avoir tué un homme.

9. Un homme, pour donner de la viande à sa famille, se change en porc sauvage et entre dans le piège qu'il a construit. Puis sa femme le mange croyant consommer de la viande de porc.

10. Pour faire croire à sa belle-mère potentielle qu'il est présent dans la hutte de guet, un chasseur se fait remplacer par un insecte qui donne la réplique à la femme. 11. Dans un village, les hommes ne connaissent pas la chaux pour consommer le bétel. À la place, ils préfèrent le sang menstruel de leurs femmes.

La liste de tels déplacements métaphoriques pourrait être longue, car c'est l'une des formes d'humour les plus fréquentes dans la littérature orale des Eri. Dans cette sélection, on a partout une substitution inattendue ou un "mauvais usage»: nourriture et éléments de parure utilisés comme armes (1), pierres chaudes devenant nourriture (3), excréments mis à la place d'aliments (2), animaux ou objets jouant le rôle de personnes $(7,8,10)$ ou au contraire homme prenant la place d'un animal en tant que nourriture de sa femme (9), pénis remplacé par un fruit urticant (6) ou traité comme une herminette (5), femme s'arrogeant des attributs masculins (4). Remarquons d'emblée que le procédé métaphorique est ici associé à l'une ou plusieurs des autres catégories retenues plus haut: choix d'un domaine privilégiant le comique (sexualité, pollution, etc.) ou situations cocasses entre personnages antagonistes. En effet, la transgression sémantique utilisée seule, au lieu de conduire au comique, pourrait tout aussi bien déboucher sur le poétique ou sur le tragique. Il lui faut l'adjonction d'un autre élément pour susciter le rire.

\section{Déplacements métonymiques}

Ce procédé est beaucoup plus rare et il s'agit toujours du type métonymique dit « la partie pour le tout». Voici d'abord un exemple simple où le comique est dû non seulement à l'image métonymique elle-même, mais aussi à l'aspect dérisoire de la " partie » sauvée des eaux :

12. Wefroog, près de se noyer dans une rivière, appelle son chien Urey à son secours.

Celui-ci nage jusqu'à lui, mais ne ramène qu'une mèche de cheveux de son maître.

17 Dans les deux illustrations qui suivent, la partie ne remplace pas le tout (comme dans 12), mais le signale, en annonçant sa présence (en l'occurrence dangereuse) :

13. Des génies femelles emballent du sagou dans des feuilles et se camouflent en cachant leur tête également dans des feuilles (pour surprendre Wefroog). Mais l'une d'elles a laissé dépasser une mèche de cheveux. Wefroog arrive: "Oh! Il y a beaucoup de sagou par ici!» Il va tâter chaque paquet: «Ah! Ce sagou est bien dur ! » Mais il découvre la mèche de cheveux, comprend l'astuce et s'enfuit...

14. Un homme travaille dans son jardin, juste à côté d'une plate-forme funéraire. Soudain, il remarque que les pieds du cadavre dépassent des enveloppes en écorces et sont maculés de boue fraîche...

Si 13 ne provoque chez les auditeurs eri que du rire et de l'amusement, l'épisode 14, lui, fait rire, mais avec une nuance de frayeur. C'est que les contextes sont dans l'un et l'autre cas très différents : les génies femelles sont censés exister, mais ne sont pas considérés comme dangereux, alors que les Eri craignent véritablement la rencontre avec les fantômes de morts récents. 
19 Ailleurs, la partie du tout n'est pas seulement un substitut passif ou un signal, mais remplace le tout complètement dans l'action ou la parole; elle redevient un être total en quelque sorte et c'est sans doute à cette invraisemblance que l'on doit le comique du passage suivant :

15. Un homme devenu cannibale en mange un autre. Il consomme le cœur en dernier. Mais le cœur dans son estomac se met à répéter tout ce qu'il dit. Le cannibale effrayé ne sait plus où se cacher. Le cœur de la victime appelle les siens à son secours...

20 Là encore, il faut reconnaître que le dispositif métonymique n'explique pas le comique à lui seul. Il suffit pour cela de rappeler que le procédé consistant à faire voir la partie annonçant la présence du tout encore caché est fréquemment utilisé dans notre " cinéma d'horreur » ou dans certains films policiers (l'ombre ou la main du meurtrier, etc.) et que l'effet produit est d'abord l'angoisse, bien que souvent mêlée au rire. Dans l'épisode 14, on a un rire teinté de frayeur. C'est le contexte et toutes les connotations culturelles qui déterminent si l'effet de surprise et le sentiment d'insolite vont tourner à l'avantage de la détente ou à celui de la crispation. Notons enfin que si la vue soudaine de la partie révélant la présence jusqu'alors insoupçonnée d'un tout dangereux ne se produisait ni dans le conte ni dans le film, mais dans la réalité, c'est dans l'effroi le plus dépourvu d'humour que nous, Eri ou Européens serions d'un coup plongés. L'imaginaire neutralise l'angoisse qui serait produite par les mêmes faits transposés dans le réel, alors que dans le récit imaginaire lui-même et à un second degré, le climat créé par le sens permet ou non à la transgression sémantique d'être perçue comme un lieu du comique.

21 Comme la métaphore, la métonymie fait donc sortir du commun, modifie l'ordre habituel des choses, brouille les catégories, transgresse les lois de la logique. C'est d'abord la surprise qui est ressentie face à cette nouvelle façon d'ordonner les faits, mais cette surprise ne peut rester neutre et, selon le contexte du récit, les autres dispositifs mis en œuvre et les connotations culturelles et inconscientes qu'elle déclenche, elle sera ressentie comme poétique, tragique, comique ou tragi-comique. Mais, malgré ces voies diverses, la transgression sémantique demeure, comme les exemples donnés ci-dessus l'illustrent, l'une des conditions possibles du comique.

\section{LE COMIQUE D'ANTAgONISME}

Ce ne sont plus ici les catégories sémantiques qui sont en cause, mais les attitudes réciproques de deux ou plusieurs personnages. Dans la transgression sémantique, on avait un élément qui changeait de catégorie (fruit $=$ arme; pierres chaudes $=$ nourriture f fruit urticant $=$ pénis, etc.). Dans le comique d'antagonisme, la transgression est d'ordre social, mais non pas sémantique. Elle concerne la réciprocité de la communication et se fonde sur une communication faussée. Si de deux personnages, l'un cherche à tromper l'autre ou que les deux tentent de se jouer des tours, on a une transgression, un déséquilibre, une réciprocité faussée qui, si le contexte et l'histoire s'y prêtent, peuvent créer un effet comique. On aura alors les situations bien connues dans notre comique théâtral du quiproquo ou du trompeur trompé. On verra plus bas que les éléments scatologiques ou sexuels se prêtent particulièrement bien à ce genre de procédé. Nous donnons ci-après pour illustration l'histoire entière de la dispute de Wefroog et des génies féminins sawango à propos des fruits du pandanus (cf. 1 et 2 ). 
16. Une sawango met une feuille tanget à un pandanus [pour en marquer la propriété]. Wefroog arrive et enlève la tanget; il en place une autre pour lui. Il s'en va et la sawango revient et remplace à son tour la tanget. Cette sawango s'appelle Tabirni. Wefroog revient et remplace encore une fois la feuille tanget. Mais Tabirni s'est cachée et attend. Wefroog vient cueillir les fruits du pandanus. Tabirni dit: «Qui a pris les fruits de mon arbre?»- «C'est moi », dit Wefroog et il lui donne un fruit vert, gardant le plus mûr pour lui. "Il n'est pas mûr ", dit la sawango, "À qui est le fruit mûr là-bas? » - «À moi », dit Wefroog. « Et celui-ci, le vert? » - « À toi »; Tabirni saisit le fruit vert et en frappe Wefroog, qui le ramasse à son tour et en frappe Tabirni. Tous les deux se battent. Wefroog ôte sa ceinture en os. La sawango en fait autant. Ils se frappent maintenant avec leurs ceintures. Wefroog, battu, s'enfuit. Tabirni ramasse les morceaux de fruits de pandanus et va les préparer.

D'entente avec Wefroog, son cousin croisé, Abunung, imite l'aboiement d'un chien à l'affût, d'un bandicoot. La sawango prend son herminette, son fils et du feu [pour enfumer le terrier] et s'en va voir. Pendant ce temps, Wefroog revient et mange les fruits préparés par Tabirni; il en emporte une partie pour Abunung. En partant, il laisse ses excréments (fruits de pandanus déjà digérés, précise le narrateur) dans la maison de la sawango. Il en met dans le filet du génie ; il en laisse partout : autour de la maison et sur le chemin; puis il s'en va. Wefroog rejoint Abunung et lui donne sa part de pandanus. Revenue chez elle, la sawango s'écrie d'étonnement: «Qui a mangé mes fruits? Il n'y a que des excréments!! » Quand elle s'approche, les mouches s'envolent par nuées des excréments de Wefroog.

Les thèmes de la duperie et du vol (l'une des formes les plus simples de transgression sociale et morale) se retrouvent dans presque tous les récits eri (voir plus haut les exemples $3,6,8,9,10,13)$, mais plus particulièrement encore dans les « histoires de fantômes ». Le fantôme est en effet une entité antisociale par définition qui vit sous terre dans une alternance inversée des jours et des nuits et remonte chez les vivants pour les voler, les tromper, les dévorer ou simplement pour s'amuser à leurs dépens. Rien d'étonnant donc à ce que la rencontre d'un vivant et d'un fantôme (qu'il faut d'ailleurs distinguer de l'«âme " du mort) tourne très vite à un concours de ruses, comme le montre le texte suivant :

17. Un homme en rencontre un autre et lui propose d'aller le lendemain pêcher ensemble. Un fantôme caché à proximité entend leur conversation. Le lendemain, le fantôme se présente sous les traits du second des hommes. Le premier, Hune, croit qu'il s'agit de son parent et part à la pêche avec le fantôme. Hune pêche, mais le fantôme va chercher d'autres fantômes; il les emballe dans des feuilles pour les camoufler. Il apporte les paquets et dit à Hune qu'il a trouvé des quantités de champignons. Il dispose les paquets tout autour de l'endroit où Hune pêche. L'oreille du fantôme se met à bouger. Hune s'en aperçoit (et comprend ainsi qu'il est en présence d'un fantôme). Il prend son arc et ses flèches et s'apprête à partir. Il dit au fantôme : «Je vais voir s'il y a un pigeon couronné là-bas ». Hune s'en va. Après un moment, le fantôme l'appelle : «Hune! Hune!» Pas de réponse. Tous les fantômes sortent alors de leurs cachettes et se mettent à appeler : «Hune hnu hnu, Hune hnu hnu... ! "Hune, rentré chez lui, se barricade dans sa maison. Les fantômes arrivent et grattent à sa porte.

Enfin, un dernier exemple de tromperie met en scène une mère ayant écarté sa fille pour se réserver les faveurs du jeune homme qu'elle désire épouser. Son comportement de «vieille» voulant jouer à être jeune, son non-respect des catégories générationnelles et de la compatibilité des âges (transgression sémantique et sociale) la rend déjà ridicule aux yeux des Eri, mais soudain la voilà trompée par l'homme et en même temps par un insecte dont le bourdonnement sans signification répond à des tentatives de séduction: 
18. Le jeune homme a construit sur un arbre une hutte de guet pour flécher les cacatoès, tandis que la femme ramasse les oiseaux tombés : un cacatoès blessé se cache dans le trou d'un crabe terrestre et la femme travaille à élargir l'ouverture pour capturer l'oiseau. Pendant ce temps, le jeune homme se fait remplacer par une guêpe mumuk dans son abri. Puis il descend et va rejoindre la fille de la vieille femme à la maison. La mère revient avec le cacatoès et s'adresse à l'homme (qu'elle croit dans la hutte) : "Y a-t-il encore des cacatoès dans les branches? " La guêpe répond : «mumumumumu». - «As-tu encore des flèches ?» - « mumumumumu». - «Veux-tu encore tirer?» - «mumumumumu». «Veux-tu que je monte pour faire l'amour?»- «mumumumumu ». [Elle grimpe finalement armée d'une herminette, avec l'intention de frapper l'homme qui se moque ainsi d'elle : la hutte est vide...].

\section{LE PROCÉDÉ NARRATIF : LE COMIQUE DE RÉPÉTITION}

ernier texte construit son comique à la fois sur la transgression, par le rôle de dupe d'un personnage déjà ridicule à travers tout son comportement, par le "dialogue » cocasse entre ce personnage et un insecte, mais aussi par la répétition des répliques de l'insecte. Le comique de répétition est à plusieurs reprises attesté dans la littérature orale des Eri, mais il diffère de celui auquel Molière nous a habitués. Chez les Eri, la répétition peut concerner une action, une situation et/ou une parole. Le dialogue absurde entre la femme et l'insecte est à la fois une répétition de situation et de langage. Mais le plus souvent l'aspect linguistique est escamoté au profit d'un comique de situation répétitif, la répétition prenant alors la forme d'une double situation alternative : deux personnages se trouvent tour à tour dans une situation donnée ou intervertissent leurs rôles respectifs (cf. début de 16 et l'épisode des étuis péniens dans 22). L'exemple qui suit met en scène la même mère que précédemment voulant rivaliser avec sa fille pour s'attirer les faveurs du jeune homme; blessé, ce dernier se fait alternativement porter par la mère et par la fille ; pour la première il se fait lourd, pour la seconde c'est une plume :

19. La mère dit à sa fille: «Va vite devant, dégage le chemin pour que je puisse passer en le portant. » La fille va devant et débroussaille le sentier. La mère suit en portant l'homme blessé : il est extrêmement lourd, elle marche pesamment. Elles arrivent à un endroit dégagé : «Huu!», la mère veut se reposer, elle dépose l'homme à terre. La fille: "C'est mon tour, je vais le porter.» La fille prend l'homme sur son dos : il est très léger et elle marche facilement. La voici maintenant qui court ! «Ah! doucement - lui crie la mère - c'est ton père adoptif, ne cours pas comme cela. Je vais le porter moi-même. » La mère s'approche et prend l'homme à nouveau. Elle veut l'emporter rapidement, mais il est lourd comme racine en terre. Elle est inondée de sueur...

Dans l'exemple ci-après, la forme répétitive est un peu différente, car elle correspond à une évolution par étapes successives qui crée, nous semble-t-il, un facteur comique dans un contexte par ailleurs dominé par l'insolite, le tout s'articulant sur une histoire de duperies entre un homme d'un côté, et sa femme et ses filles de l'autre :

20. Un homme a tué un porc sauvage et un casoar. Il les laisse pourrir sur une plateforme funéraire en forêt. Il n'apporte à sa femme et à ses deux filles que des lézards, etc. La femme méfiante envoie la cadette de ses filles en forêt et celle-ci découvre le porc et le casoar. La mère et ses filles décident de tuer l'homme. Elles se transforment en porcs sauvages et attaquent le chasseur. Il reste inanimé. Elles l'enterrent. Elles rentrent chez elles et rapportent le porc et le casoar. Elles les mangent. Pendant le repas, la mère envoie à nouveau sa fille voir en forêt. La fille revient et dit que les cheveux de son père dépassent du sol. Plus tard, la mère envoie encore sa fille. Celle-ci revient et dit qu'on voit maintenant le front de son 
père qui dépasse. Plus tard, elle repart voir. Elle revient et dit qu'on peut voir ses yeux. Elle repart encore, revient et dit que son nez est hors du sol. Elle va encore voir, revient et dit que sa bouche est visible. Elle retourne encore : toute la tête est sortie du sol. Elle repart: les épaules sont dehors... puis la poitrine, les hanches. Enfin, la fille vient dire que son père est maintenant complètement sorti de terre et qu'il est assis sur le sol. Puis elle vient annoncer qu'il est allé cueillir des feuilles médicinales. Les femmes ont terminé leur repas. La fille annonce alors que son père vient...

\section{LES TERRAINS PROPICES À LA TRANSgRESSION}

27 À l'examen des textes eri, nous nous sommes aperçu que le comique se greffait de préférence sur des histoires ayant pour thème général l'un des quatre sujets suivants : relations de parenté, sexualité, pollution, alimentation. Ce choix ne constitue évidemment pas en soi un procédé comique; il offre seulement à l'humour un terrain favorable. Ce terrain correspond à des domaines réglés par un certain nombre de contraintes ou d'interdits et au sein desquels les relations aux choses ou aux êtres doivent se conformer à des normes strictes. Les transgresser soi-même dans le réel conduirait au conflit, à la crise ; mais les faire transgresser par des personnages imaginaires peut déboucher sur le comique et libérer quelque peu l'inconscient.

On sait que dans les sociétés non industrielles la parenté joue un rôle social capital et que les relations parentales sont réglées de façon souvent très formalisée. Un individu qui dans la réalité ne se conformerait pas à ces normes scandaliserait. Dans la littérature orale, il amuse. C'est le cas de la mère déjà âgée qui se comporte comme une jeune auprès du prétendant de sa fille : elle refuse son statut de mère et de femme d'âge mûr et recherche un mariage avec un homme trop jeune pour elle. Or, les Eri veillent toujours dans leurs pratiques matrimoniales à ce que les conjoints aient à peu près le même âge. La mère cherche en outre à instaurer, pour parvenir à ses fins, une relation de parenté imaginaire, entre le jeune homme et sa fille: «Ne le touche pas, c'est ton père adoptif (ou " beau-père ", c'est-à-dire le nouveau mari de la mère veuve), répète-telle à plusieurs reprises à sa fille. La confusion par la mère des catégories parentales s'ajoute à celle des groupes d'âge. En outre, la vieillesse qui veut paraître jeune est un thème comique universel; plus loin dans le même récit :

21. La mère, à la recherche de sa fille enlevée par le jeune homme, rencontre le génie des jardins qui incarne le rythme cyclique de l'horticulture itinérante, et se présente à elle d'abord très vieux pour symboliser la jachère, puis le lendemain jeune et beau, symbole de l'essart défriché et brûlé prêt à de nouvelles plantations. La femme veut l'imiter et va stupidement harceler un essaim de guêpes dont les piqûres la font enfler, tendent sa peau et effacent ses rides. Ainsi « rajeunie », elle se présente au génie, qui n'est pas dupe...

Les rapports entre époux sont souvent dans la littérature eri l'objet de transgression créant des situations burlesques: la femme préparant des champignons sur lesquels son mari a déféqué et les lui donnant à manger (cf. 25), le mari (et père) transgressant son devoir de chasseur nourricier et traitant le cochon et le casoar tués comme des cadavres humains (cf. 20), le même homme tuant ensuite sa femme et en consommant les seins en guise de champignons, etc.

La sexualité est aussi dans la littérature eri un prétexte fréquent à déclencher le rire. Plusieurs courtes illustrations en ont déjà été données dans les exemples cités. En voici deux exemples plus longs : 
22. Abunung a dérobé le serpent que des enfants avaient mis à cuire dans le feu, il l'a mangé et est allé se reposer sur un arbre : pour se venger, les enfants récoltent des épines et des insectes venimeux et vont les placer sur le pénis d'Abunung. Les bestioles se glissent sous son prépuce. Les enfants partis, Abunung se réveille et va chez Wefroog lui proposer une danse avec les étuis péniens cérémoniels [cette danse consiste à faire se balancer l'étui vers le haut afin qu'il frappe une ceinture en os portée sous l'abdomen]. Tous deux dansent. L'étui pénien de Wefroog sonne bien: gwan gwan gwan! Mais l'étui d'Abunung fait un bruit sourd. Abunung propose à Wefroog d'échanger leurs étuis péniens. Mais c'est toujours celui que porte Abunung qui sonne mal. Wefroog demande alors à sa femme de maintenir sa vulve ouverte à l'aide de deux bâtonnets, pour que le pénis d'Abunung se dresse. La femme est en train de préparer la gelée de sagou; elle s'exécute et Wefroog part en forêt. Le pénis d'Abunung se dresse, son étui pénien se brise en petits morceaux et, tout autour d'eux, les sagoutiers, les palmiers de toutes sortes laissent pendre leurs palmes et cessent de croître ; tous les animaux se taisent soudain. Pendant ce temps, la femme de Wefroog continue à emballer le sagou préparé. (Avec la semence) insectes et épines sont évacués et se répandent sur le sagou. Alors tous les sagoutiers et les palmiers de toutes sortes se remettent à croître et dressent leurs palmes, tandis que les oiseaux se remettent à chanter. Wefroog en forêt remarque que tout s'est tu, puis que tout revit à nouveau. Il pense : « Ah ! C'est bien, Abunung a terminé. » Il revient.

Le mélange de la semence au sagou est évidemment une transgression, mais exprime ici en même temps une relation symbolique extrêmement importante dans la pensée eri et fait allusion à un rite bien précis d'une cérémonie de fertilité. C'est un bon exemple de désacralisation par le comique. Dans le texte suivant, il n'y a pas transgression à proprement parler, mais anomalie - ce qui revient un peu au même. Si la femme sans sexe fait déjà sourire en soi, la technique utilisée par son beau-père pour lui en ouvrir un donne lieu à un comique d'antagonisme, mais aussi à une forme amusante de déplacement métaphorique qui évoque lui-même des rapports symboliques bien précis : fruit du Piper betle = clitoris ; jus de la chique de bétel = sang menstruel ; et, d'une façon plus générale, mélange du bétel = sexualité.

23. À cette époque, il n'y avait pas de forêt, mais seulement de la savane. Un vieillard, Wefroog, vit avec son fils et sa fille. Il dit au garçon : «Épouse ta sœur. » Le fils refuse: "Je ne suis pas un animal." «Eh! bien, dit le père, pars ailleurs échanger ta sœur contre une épouse. » Le fils part à Kumwaag, échange sa sœur et revient avec une épouse. Le jeune couple va en brousse pour s'accoupler, mais il n'y parvient pas, car la femme n'a pas de vagin. Wefroog se plaint que sa bru ne lui prépare rien à manger. Il lui demande de cueillir pour lui des noix d'arec : « Monte à l'arbre, lance-moi des noix, puis laisse-toi tomber à terre le long du tronc.» Pendant que la femme est au haut de l'aréquier, il plante un bâton muni d'une pierre tranchante au pied de l'arbre. En se laissant retomber, la bru s'empale et la pierre lui ouvre un vagin. Avant qu'elle ne se relève de sa chute, Wefroog [qui chique déjà les noix qu'elle lui a lancées] lui crache dans le sexe sa salive rouge et y lance un fruit de Piper betle. Ensuite, le couple veut à nouveau faire l'amour. Tous deux vont en brousse, mais ils renoncent, car il n'y a pas de forêt et Wefroog pourrait les voir. La femme mécontente se plaint à son beau-père. Wefroog, pendant la nuit, plante les poils de sa barbe puis fait souffler un grand vent qui porte les poils au loin. Sa bru a peur du vent et se réfugie près de Wefroog. Celui-ci fait cesser le vent. Le matin, la forêt s'étend partout. Les époux repartent. À une certaine distance, ils se retournent, regardent autour d'eux, mais ne voient plus la maison de Wefroog. Ils font l'amour. La femme revient contente et prépare un bon repas à son beau-père.

Comme la sexualité, le thème de la pollution fournit au comique un terrain favorable et en particulier la pollution excrémentielle. La littérature eri en a fait principalement soit un moyen de souillure externe, soit un aliment involontaire. On peut dire que, de 
manière générale, être souillé d'excréments c'est d'abord être ridicule, et plus la matière est fraîche et salissante, plus importante est la souillure, plus grande sont la gêne de la personne souillée et le rire (même intériorisé) de l'observateur. Chez les Eri, il en va de même, mais ce n'est que dans l'imaginaire des aventures de Wefroog avec les génies féminins que la souillure n'est plus seulement un accident, mais un moyen prémédité de confondre l'adversaire :

24. Poursuivi par les esprits femelles sawango, Wefroog s'enfuit en laissant à tous moments ses excréments sur le chemin. Il parvient au sommet d'une colline rocheuse d'où il fait rouler un bloc de rocher sur les divinités. Les unes meurent, les autres sont seulement projetées au sol. Toutes restent inanimées : «Wefroog mêle d'abord des excréments à leur sagou, leur viande de porc, etc. » Puis il ramasse une baguette de Pometia pinnata. Il s'approche de chacune d'elles et les pique dans l'œil. Quand l'œil ne réagit pas, c'est qu'elles sont évanouies : il verse alors dans l'œil des excréments liquides [littéralement "diarrhée »]. Il en pique ensuite une autre, son œil ne réagit pas, il y verse des excréments liquides. Il continue ainsi pour chaque sawango. Il en reste maintenant deux: le déplacement d'air de la roche les a projetées à l'écart. Wefroog se penche vers chacune d'elles pour leur piquer l'œil : il pique, pique, pique, leurs yeux se ferment. Il dit [pour faire croire qu'il les croit mortes] : « Ces deux-là sont aussi mortes. » À distance, il fait semblant de leur verser des excréments dans l'œil. Puis il s'enfuit effrayé...

Le fait que cette particularité d'utiliser les matières fécales comme moyen de souiller l'ennemi soit, dans les mythes eri, essentiellement réservé à Wefroog, héros civilisateur et trickster, mais par ailleurs considéré comme bon, faible, mobile et créateur, est un moyen de le rendre en outre comique, ce qui nous introduirait à une nouvelle catégorie : le comique de caractère, analysé par Bergson.

Mais le comble de la souillure scatologique est la scatophagie. Celle-ci n'est, le plus souvent, que suggérée, lorsque des excréments sont mêlés à de la nourriture (cf. 24) ou lorsqu'ils la remplacent (cf. 2, 16) sans qu'il y ait consommation effective. Plus rarement un personnage consomme des matières fécales et, dans l'exemple ci-dessous, il s'agit de ses propres excréments. L'auto-scatophagie, en tant que forme d'autocannibalisme doublé de souillure, apparait ici comme un cumul de transgressions et de ridicule :

25. Un homme va déféquer sur un tronc d'arbre abattu sur lequel poussent des champignons. Sa femme cueille ensuite ces champignons, les prépare, puis les donne à son mari. Tandis qu'il mange, elle lui demande : « Qui a déféqué sur le tronc là-bas? »- «C'est moi », répond-il. «Eh! bien, tu ne fais que manger tes propres excréments.»

La souillure n'est pas toujours excrémentielle et peut être le résultat d'un contact avec le sang menstruel, objet de tabous sévères presque partout en Nouvelle-Guinée (cf. aussi 11$)^{2}$ :

26. Abunung veut épouser Oogango, la veuve de Wefroog qu'il a tué. Elle parvient à s'enfuir de la maison en lui jouant un mauvais tour. Abunung suit ses empreintes qui le conduisent au pied d'un grand arbre. Il s'assied pour se reposer et sent quelque chose qui tombe sur son dos, il s'essuie de la main et regarde: «Qu'est-ce que c'est? » Il hume le liquide : «Oh ! un oiseau sans doute. » À nouveau, il sent quelque chose tomber sur lui ; il s'essuie, respire puis goûte au liquide; le goût fort lui fait lever la tête : il voit Oogango assise sur une branche, elle a ses règles... [Suit une scène où Oogango laisse pendre son très long clitoris, Abunung s'y agrippe, elle le hisse ainsi, mais à chaque fois il retombe brutalement sur le sol les quatre fers en l'air. Finalement, trop excité à la vue d'Oogango, il se met à forniquer dans les trous qu'il trouve dans le sol et le tronc des arbres alentour]. 
Toutes les formes de comique sont réunies dans cette séquence : transgression par la consommation de l'inconsommable par excellence (notons qu'on y vient par étapes : toucher, odorat, goût), donc souillure grave, répétition de la chute d'Abunung, comique de situation inscrit, au niveau du sens, dans le contexte de rapports hommes/femmes inversé (une femme se moque d'un homme et le ridiculise) et d'une sexualité désordonnée: les temps primordiaux étaient marqués par une fécondité extrême et une sexualité excessive (organes démesurés) et pas encore socialement sanctionnée. L'épisode du sang humé puis goûté appartient déjà à une version secrète du mythe et est escamoté par le narrateur s'adressant à un auditoire jeune. Le rapport au sacré y est trop fort par ailleurs pour que la situation devienne ouvertement comique; contrairement à la relation de l'acte scatophagique qui peut autoriser la libération du rire, l'allusion à la consommation du sang menstruel impose le contrôle des émotions et interdit les éclats : le comique est bloqué par le sacré et demeure non réalisé, car ce n'est pas le rire qui est retenu pour des questions de bienséance, mais le comique même qui contrarié par le sacré, n'est plus ressenti pleinement comme tel. Les références culturelles auxquelles renvoient ces deux matières, excréments et sang menstruel, sont seules responsables du contraste entre extériorisation et intériorisation du rire face à l'acte de leur consommation: la première substance n'est en effet qu'un déchet irrécupérable dont on doit se débarrasser définitivement, alors que la seconde est pour les Eri la part féminine qui, dans l'acte procréateur, vient s'allier à la semence mâle pour constituer l'embryon; c'est donc une matière puissante et dangereuse qui est d'ailleurs récupérée à des fins rituelles. Au caractère sacré de la seconde s'oppose l'aspect dérisoire de la première, un peu comme à la puissance procréatrice de la femme s'opposerait la dérision de l'homme auquel échappe le pouvoir de grossesse et qui n'aurait que ses excréments pour polluer ses adversaires féminines.

L'anthropophagie, un aspect particulier de la transgression alimentaire (les Eri n'ont jamais pratiqué le cannibalisme), est aussi un champ où fleurit volontiers l'humour. La chair humaine est l'aliment de certains personnages mythiques, des esprits féminins de la forêt ou des fantômes, la caractéristique de ces derniers, notamment, étant de se nourrir de l'inconsommable et d'appartenir à un univers inversé (donc à un monde de transgression).

Le rire est universellement partagé, bien que son intensité ou son degré de spontanéité varie d'une culture à l'autre. Et lorsque l'étranger ne comprend pas les motivations du comique dans un épisode ou une situation donnée, ce n'est pas parce que la logique du comique de ses hôtes est différente de la sienne, c'est seulement qu'il lui manque des données culturelles ou qu'il n'est pas sensibilisé à la culture qu'il découvre. Cette barrière de la spécificité culturelle franchie, le rire devient un bien universel tant dans ses articulations sémantiques que dans sa nature neuropsychologique. L'exemple de cette société de Nouvelle-Guinée, où les mécanismes du rire sont en gros les mêmes que ceux suscités par notre théâtre ou notre cinéma et pourraient presque tous être classés selon les catégories proposées par Bergson ${ }^{3}$, nous semble bien mettre en évidence l'universalité formelle du comique. Au-delà des contenus variables que seule une approche culturaliste peut appréhender pleinement, ces motivations formelles du rire correspondent donc à une faculté de l'esprit humain à réagir de façon invariante à 
certains types de stimuli. Ces derniers, on l'a vu, sont des structures sémantiques. Leurs caractéristiques formelles font qu'elles fonctionnent indépendamment du contenu narratif, mais on a vu qu'elles assurent mieux le comique lorsqu'elles se greffent sur certains terrains thématiques. Et le fait que ces thèmes nous soient eux-mêmes apparus comme universellement propres à favoriser le comique, à cause des interdits moraux qui les gouvernent dans presque toutes les sociétés humaines, vient corroborer notre argument universaliste. Si différente que soit la culture eri par rapport à la nôtre, et si étrangers les thèmes de leur littérature orale, cela n'enlève rien à l'accessibilité de leur comique. Et si, au cours d'un récit, les auditeurs locaux s'esclaffent alors que l'étranger ne fait parfois que sourire poliment, c'est davantage à cause d'une différence de degré dans une suggestibilité culturellement déterminée que par l'effet d'un écart structurel dans les articulations de l'humour.

\section{BIBLIOGRAPHIE}

BERgson, Henri, 1900, Le Rire : essai sur la signification du comique, Paris, Presses Universitaires de France.

CLASTRES, Pierre, 1967, De quoi rient les Indiens ?, Les Temps modernes, 253, p. 2179-2198.

Clastres, Pierre, 1974, La Société contre l'État, Paris, Éd. de Minuit, pp. 113-132.

CoYAUD, Maurice, 1980, La transgression des bienséances dans la littérature orale, Critique, 394, p. 325-332.

Douglas, Mary, 1968, The Social Control of Cognition: some Factors in Joke Perception, Man, 3 (3), pp. 361-376.

Douglas, Mary, 1975, Implicit Meanings. Essays in Anthropology, "Jokes", London, Routledge \& Kegan Paul, pp. 90-114.

LEDENT, Roger, 1974, Comprendre la sémantique, Verviers (Belgique), Marabout-Université.

MoRIN, Violette, 1966, L'histoire drôle, Communications, 8, pp. 102-119.

\section{NOTES}

1. Les extraits donnés ici proviennent principalement des Eri septentrionaux et plus particulièrement de la tribu jafar.

2. Hors de la littérature, l'homme qualifié de heifu, c'est-à-dire contaminé par le sang menstruel, peut subir un rite de purification au cours duquel les enfants du village se moquent conventionnellement de lui.

3. Pour les commentaires sur l'humour selon Bergson ou selon Freud, voir M. Douglas (1968). 


\section{RÉSUMÉS}

L'humour, apparaissant de façon diffuse dans presque tous les textes de tradition orale des Eri septentrionaux (Province de West Sepik, Papouasie Nouvelle-Guinée), ne peut se donner libre cours qu'à la condition d'une désacralisation du récit. Il peut prendre trois formes différentes: comique d'antagonisme, comique de répétition et surtout transgression sémantique. Ces formes ont en outre besoin d'un terrain propice à la transgression et au comique, d'où la délimitation de quatre champs culturels privilégiés : la parenté, la sexualité, l'idée de pollution et l'alimentation.

Humor, which crosscuts nearly all the oral texts of the northern Eri (West Sepik Province, Papua New Guinea), cannot be given free expression without de-sanctifying the narrative. It can assume three different forms: comedy of antagonism, comedy of repetition, and above all, semantic transgression. Moreover, these forms need fertile grounds that favor transgression and comedy; four cultural domains appear to be privileged: kinship, sexuality, pollution and food.

\section{INDEX}

Thèmes : anthropologie (Océanie)

Index géographique: Papouasie Nouvelle-Guinée

nomsmotscles Eri

Mots-clés : comique, transgression

Keywords : Papua New Guinea, Transgression, Comedy, Anthropology 